\title{
Daan Frenkel - An entropic career
}

Robert Evans $\mathbb{C}^{\mathrm{a}}$, Amparo Galindo $\mathbb{C}^{\mathrm{b}}{ }^{\mathrm{b}}$, George Jackson $\mathbb{C}^{\mathrm{b}}$, Ruth Lynden-Bell ${ }^{\mathrm{c}}$ and Benjamin Rotenberg (1)d

${ }^{a}$ H. H. Wills Physics Laboratory, University of Bristol, Tyndall Avenue, Bristol BS8 1TL, UK; ${ }^{b}$ Department of Chemical Engineering, Imperial College London, South Kensington Campus, London SW7 2AZ, UK ; ' University Chemical Laboratory, Cambridge University, Lensfield Road, Cambridge CB2 1EW, UK ; dPhysicochimie des Electrolytes et Nanosystèmes Interfaciaux, Sorbonne Université, CNRS, PHENIX, Paris F-75005, France

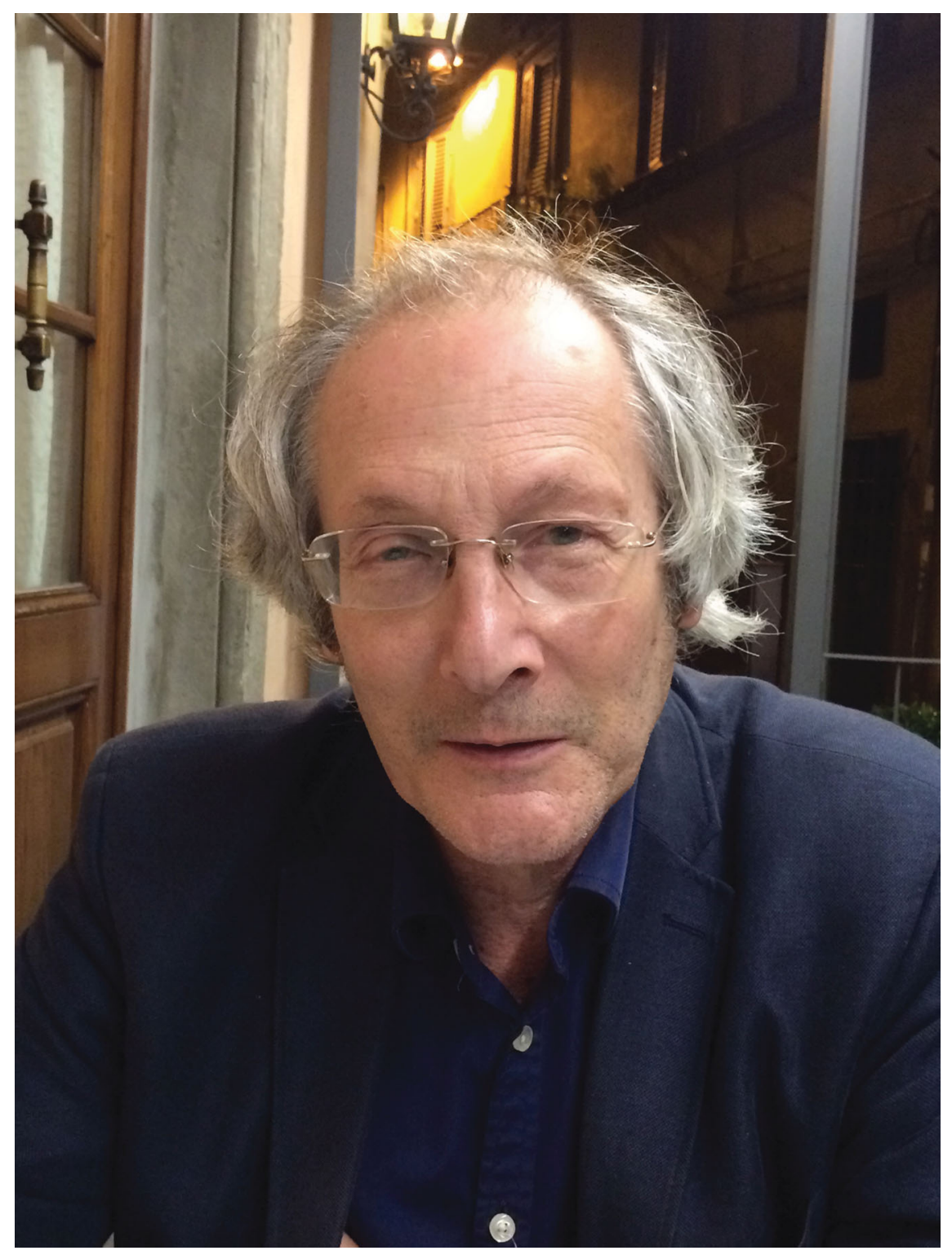

Figure 1. Daan Frenkel. 
The editors are very pleased to be able to celebrate the 70th birthday of Daan Frenkel (Figure 1) with this Special Issue of Molecular Physics. The large number and the quality of the scientific contributions, accompanied by heartfelt acknowledgements to Daan, reflect his influence on a very broad field ranging across fundamental statistical thermodynamics, computer simulation, and phase transitions. Moreover, these attest to his continuing influence on current practitioners. This is testament to the enormous respect and admiration in which Daan is held, not only for his seminal and world-leading scientific publications, but also as a true gentleman of science, friend, and colleague.

Daan's research has focused on the numerical simulation of many-body systems, with a special emphasis on problems relating to ordering and self-assembly in soft matter. Daan has developed novel Monte Carlo algorithms for free-energy calculations and for the simulation of chain molecules. In addition, he has established popular and widely employed techniques to compute the various types of disordered packings of jammed particles. Applications of his research lie in the areas of liquid-crystalline ordering, crystal nucleation, and complex self-assembly through to design rules for DNA coated nanoparticles.

Daan was born in Amsterdam in 1948, the second of four children of Maurits Frenkel and Herta Tietz (both medical doctors), who managed to escape deportation during the war. Daan's early career plans included icecream vendor (one customer only), steam-engine driver, astronomer, and archaeologist. Although he never lost interest in any of these activities, he chose to study (Physical) Chemistry at the University of Amsterdam, which encompasses his passions. As an undergraduate student (1966-1972) he attended guest lectures on computer simulation methodologies by Berni Alder and Les Woodcock.

Daan was awarded his $\mathrm{PhD}$ in experimental Physical Chemistry from the University of Amsterdam in 1977, supervised by Jan van der Elsken, where he employed infrared spectroscopy to study the molecular dynamics of dense fluids. As a young and enthusiastic $\mathrm{PhD}$ student, he was fortunate to spent five months at CECAM (then at Orsay) where he familiarised himself with molecular-dynamics and Monte Carlo simulations, meeting (amongst others) Giovanni Ciccotti, Gianni Jacucci, Mike Klein, and Aneesur Rahman. This research visit moulded his future scientific career along the path of statistical mechanics and molecular simulation.

Subsequently, Daan worked as a postdoctoral research fellow in Chemistry at the University of California at Los Angeles with John McTague and Dan Kivelson. After a short spell at Shell Research (Amsterdam), Daan was appointed to the faculty at Utrecht University. In 1987 he joined the FOM Institute for Atomic and Molecular Physics (AMOLF), holding associated professorships in Utrecht and Amsterdam Universities. In 2007 Daan was elected 1968 Professor of Theoretical Chemistry at the University of Cambridge, following on from JeanPierre Hansen, and served as Head of the Chemistry Department from 2011 to 2015.

Daan's awards are numerous. He was elected a member of the Royal Netherlands Academy of Arts and Sciences in 1998, Foreign Member of the Royal Society in 2006, Foreign Honorary Member of the American Academy of Arts and Sciences in 2008, Foreign Associate of the National Academy of Sciences (USA) in 2016, and is a Member of the Academia Europeae. Daan has received numerous international prizes, including the NWO Spinoza Award (2000), the Aneesur Rahman Prize from the American Physical Society (2007), the Berni Alder CECAM prize of the EPS (2007), and the Boltzmann Medal (IUPAP) for Statistical Physics (2016). He was awarded an honorary Doctorate from the University of Edinburgh and Honorary/Concurrent Professorships from Beijing University of Chemical Technology and Nanjing University.

In 1986, Daan married Alida Bolliger. He has two daughters (Floor and Evelien). Alida died of cancer in 1995, when their daughters were 5 and 8 respectively. In 2004, he married Erika Eiser, who now works at the Cavendish Laboratory in Cambridge.

As we have mentioned, Daan continues to play a towering role in the development of modern computer simulation and statistical mechanics of complex fluids and materials. He is renowned internationally for his pioneering contributions to understanding entropydriven phase transitions, the nature of liquid-crystalline phase transitions, freezing and glass transitions, nucleation, and for key methodological advances in computersmulation techniques, including the determination of the free energy of solid phases and the sampling of chain and polymeric molecules with biasing techniques.

Daan's long-standing interest in molecular dynamics, freezing and liquid-crystalline behaviour was kindled $[1,2]$ during his postdoctoral research at UCLA. In the mid-1980s Daan published the first molecular simulations of purely entropy-driven liquid-crystalline phase transitions: the nematic phase formed by discotic particles [3], and the smectic layered structures formed by rod particles $[4,5]$. The idea that the isotropic-nematic transition is entropically driven goes back to Onsager in the 1940s. (In 1957 Alder and Wainwright had already highlighted the importance of entropy in ordering transitions by showing that a system of purely repulsive (hard) spheres can form a solid phase, without the need 
(a)
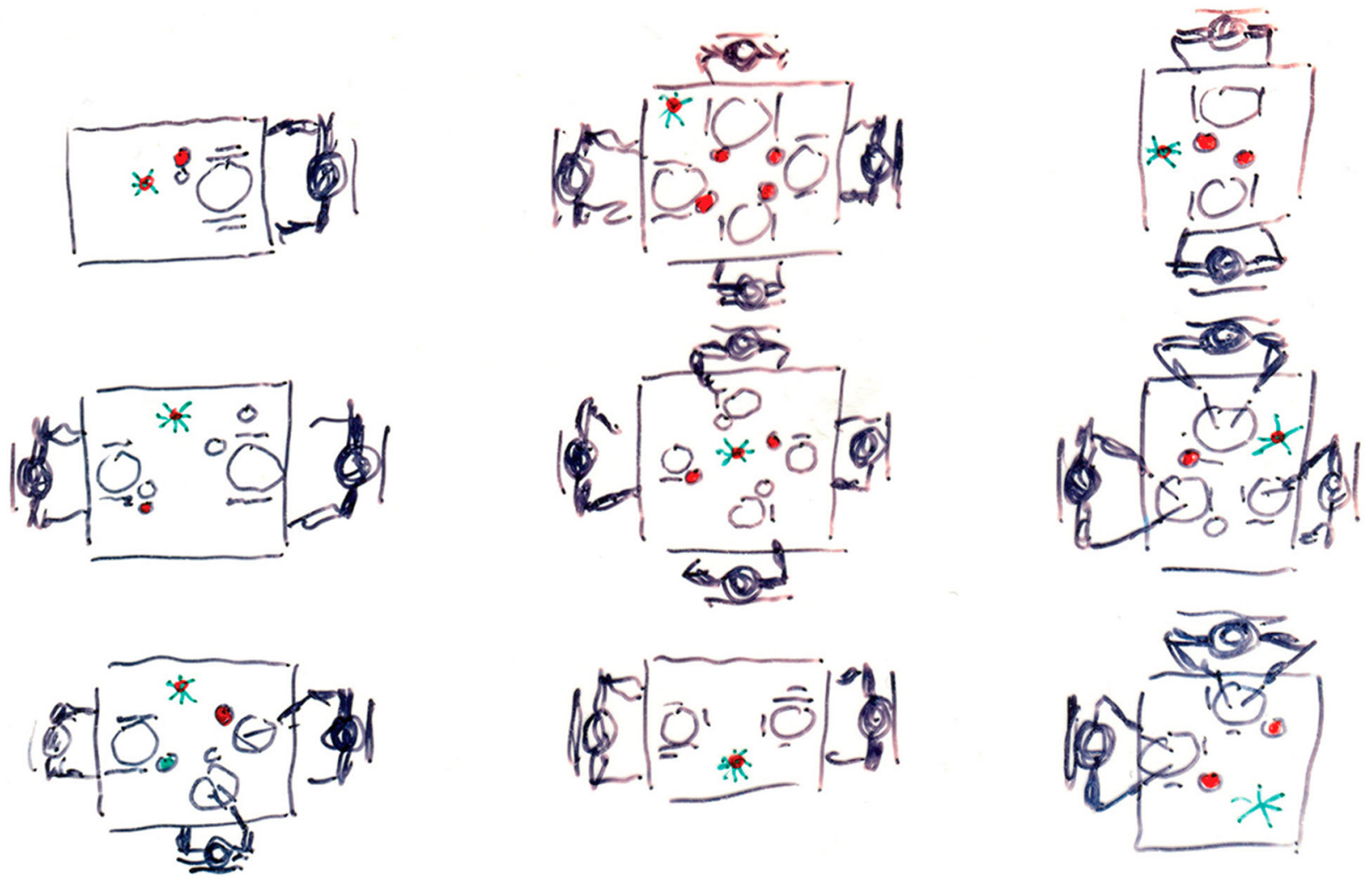

(b)

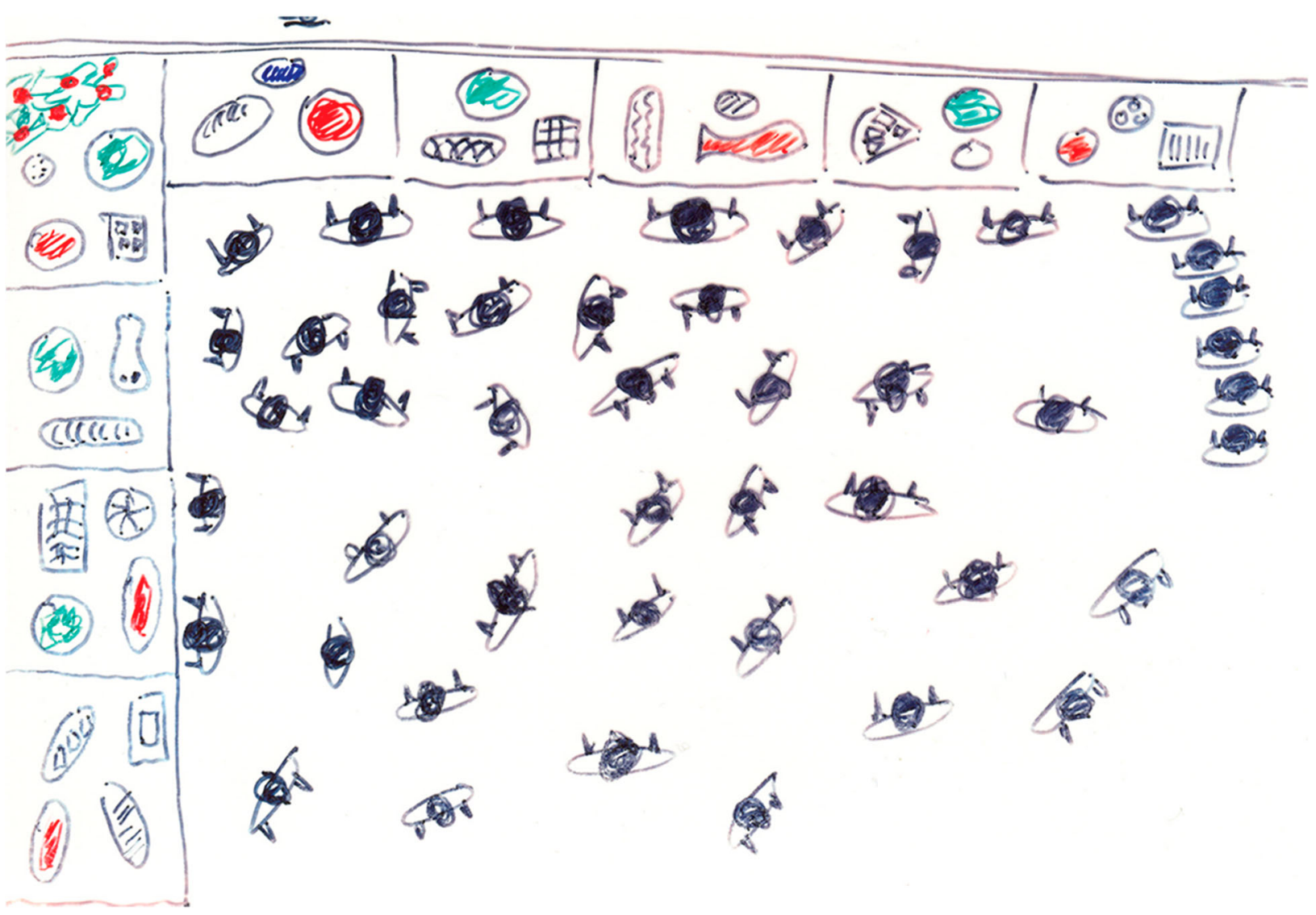

Figure 2. Daan's splendid 'dinner party' cartoon on entropy-driven phase transitions in mixtures of different sized/shaped particles: a) a low-density mixed state where the diners and tables are distributed evenly in space; b) a high-density demixed state where the diners and tables are separated to allow more free space and movement for the dinner guests. 
to invoke attractive interactions.) Surprisingly, one had to wait more than a third of a century for Onsager's clairvoyant hypothesis of the entropic role in liquidcrystalline ordering to be confirmed unequivocally and elegantly by Frenkel and co-workers. The role played by entropy in the phase separation of particles of different size and/or shape is beautifully illustrated in Daan's cartoon reproduced in Figure 2. This is but one example of his numerous cartoons designed to convey profound physical insights, often spiced with his sharp sense of humour (who could forget one of Daan's after dinner talks?).

In the mid-1980s, Frenkel and Ladd [6] developed a thermodynamic-integration molecular-simulation technique to determine the free energy of arbitrary solid phases: their methodology remains the method of choice for studies of phase transitions in solid materials and the determination of solvation properties. Amongst other notable achievements, too numerous to list, Daan's period in FOM led to: the development of a configurational bias Monte Carlo sampling scheme to determine the chemical potential of flexible chains [7]; the numerical computation of nucleation rates of crystalline structures encompassing an ingenious analysis of protein crystallization [8-11]; and the simulation of the fluidphase separation of colloid-polymer mixtures [12] and patchy colloids [13].

The 1st edition of Daan's book, with Berend Smit, 'Understanding molecular simulation: from algorithms to applications', was published in 1996. Now in its second edition, this remains a definitive text in the area; it has been translated into Chinese and Russian and has been cited many thousands of times.

When Daan moved to Cambridge his attention turned to new avenues, especially biomolecular research. Topics include: the simulation of rare events [14], aggregation and nucleation of DNA [15-18], the curvature of biological membranes [19], receptor nanoparticles [20,21], granular entropy and jamming [22,23], and phoretic effects [24].

Daan's international reputation in the simulation and theory of complex fluids and materials is reflected in a commentary accompanying the award of the Boltzmann Medal: 'He (Frenkel) is arguably the most creative and diverse "simulator" of soft matter and the self-assembly of complex macromolecular systems of his generation.' Very few would argue with this assessment. Daan has published more than 450 papers, including many seminal articles in Molecular Physics. Daan's career is faithful to his beloved entropy: he makes sense of (and quantifies) a wide range of simple and complex phenomena, he is extensive, and he rises to even higher levels. However, it is to the benefit of the community that Daan's scientific production rate does not follow the tendency of the entropy production rate to reach a minimum.

The Editors and Advisory Board of Molecular Physics are very grateful to Daan for his lasting contributions to the journal, both as an author and longstanding member of the advisory and editorial boards. We are delighted and honoured to introduce this Special Issue of Molecular Physics celebrating his scientific achievements.

\section{Disclosure statement}

No potential conflict of interest was reported by the authors.

\section{ORCID}

Robert Evans (D) http://orcid.org/0000-0002-6143-2160

Amparo Galindo (D) http://orcid.org/0000-0002-4902-4156

George Jackson (D) http://orcid.org/0000-0002-8029-8868

Benjamin Rotenberg (D) http://orcid.org/0000-0001-5198-4650

\section{References}

[1] D. Frenkel and J.P. McTague, Phys. Rev. Lett. 42, 1633 (1979).

[2] D. Frenkel and J.P. McTague, J. Chem. Phys. 72, 2801 (1980).

[3] R. Eppenga and D. Frenkel, Mol. Phys. 52, 1303 (1984).

[4] A. Stroobants, H.N.W. Lekkerkerker, and D. Frenkel, Phys. Rev. A. 36, 2929 (1987).

[5] D. Frenkel, H.N.W. Lekkerkerker, and A. Stroobants, Nature. 332, 822 (1988).

[6] D. Frenkel and A.J.C. Ladd, J. Chem. Phys. 81, 3188 (1984).

[7] J.I. Siepmann and D. Frenkel, Mol. Phys. 75, 59 (1992).

[8] J.S. van Duijneveldt and D. Frenkel, J. Chem. Phys. 96, 4655 (1992).

[9] P.R. ten Wolde, M.J. Ruiz-Montero, and D. Frenkel, J. Chem. Phys. 104, 9932 (1996).

[10] P.R. ten Wolde and D. Frenkel, Science. 277, 1975 (1997).

[11] S. Auer and D. Frenkel, Nature. 409, 1020 (2001).

[12] E.J. Meijer, D. Frenkel, J. Chem. Phys 100, 6873 (1994).

[13] N. Kern and D. Frenkel, J. Chem. Phys. 118, 9882 (2003)

[14] R.J Allen, D. Frenkel, and P.R. ten Wolde, J. Chem. Phys. 124, 024102 (2006).

[15] D.B. Lukatsky, B.M. Mulder, and D. Frenkel, J. Phys. Condens. Matt. 18, S567 (2006).

[16] P. Varilly, S. Angioletti-Uberti, B.M. Mognetti, and D. Frenkel, J. Chem. Phys. 137, 094108 (2012).

[17] A. Reinhardt and D. Frenkel, Phys. Rev. Lett. 112, 238103 (2014).

[18] S. Angioletti-Uberti, P. Varilly, B.M. Mognetti, and D. Frenkel, Phys. Rev. Lett. 113, 128303 (2014).

[19] R.N. Frese, J.C. Pàmies, J.D. Olsen, S. Bahatyrova, C.D. van der Weij-de, T.J. Aartsma, C. Otto, C.N. Hunter, D. Frenkel, and R. van Grondelle, Biophys. J. 94, 640 (2008).

[20] F.J. Martinez-Veracoechea, D Frenkel, Proc. Natl. Acad. Sci. USA 108, 10963 (2011). 
[21] R. Vácha, F.J. Martinez-Veracoechea, and D. Frenkel, Nano Lett. 11, 5391 (2011).

[22] D. Asenjo, F. Paillusson, and D. Frenkel, Phys. Rev. Lett. 112, 098002 (2014).
[23] S. Martiniani, K.J. Schrenk, K. Ramola, B. Chakraborty, and D. Frenkel, Nat Phys. 13, 848 (2017).

[24] J. Burelbach, D. Frenkel, I. Pagonabarraga, and E. Eiser, Eur. Phys. J. E. 41, 7 (2018). 